\title{
Measurement of multiparametric quantities at perception of sensory information by living creatures
}

\author{
Roald Taymanov and Kseniia Sapozhnikova ${ }^{a}$ \\ D.I. Mendeleyev Institute for Metrology, 19000519 Moskovsky pr., St.Petersburg, Russian Federation
}

\begin{abstract}
In the paper it is shown that at present the problem of measurements of emotions as multidimensional (multiparametric) quantities is of interest. A measurement model developed by the authors is presented. It enables signals-stimuli generating emotions in sound combinations and their series to be selected and quantitatively estimated. The measurement model provides an adjusted delay (short-time memory) and joint non-linear conversion of audible and delayed acoustical signals, selection of infrasound signals-stimuli as well as identification of emotional images including the ensembles of signals-stimuli and their series. It is demonstrated how the "mechanism" of emotional perception of sound impacts was developed in the process of evolution. Possibilities to decode the emotional content of biolinguistic signals of animals are considered. Future trends of investigations are indicated.
\end{abstract}

\section{Introduction}

The analysis of publications in various branches of science $[1,2]$ demonstrates that the interest in measurements of quantities related to man, his memory, talents, level of knowledge, etc. grows significantly faster than that in measurements related to inanimate nature. At the same time there is a growing interest in the investigations concerning interaction of people with each other.

The utility of investigations in this field is proved by the appearance of a number of scientific conceptions in which the role of psychic peculiarities of separate social groups is accentuated under the circumstances when consumers are forming decisions with regard to explicit costs. On the basis of one of such conceptions, the bank business of the "father of microfinancing," Muhammad Unus, Nobel Prize laureate in 2006, and his followers is being successfully developed, whereas other businessmen are making good use of the conception for developing their neuro-marketing (advertising) business.

Another confirmation of the utility can be the results of psycho-diagnostics and art-therapy. The latter applies various effects over an emotional sphere of patients. Increasing the interest to these spheres is natural on the background of an increasing number of publications, informing readers about undesirable consequences of a pharmaceutical composition intake. A list of examples can be continued..

When the matter concerns the perception of sensory information by a human or some other living creatures, in the majority of cases the quantities of interest for investigations are multidimensional (multiparametric). To make a transfer to their measurement it is necessary to ground and develop corresponding measurement models. The measurement model in biology, psychology, economics or other sciences related to animals, humans and their activities, as a rule, reflects the idea of a model implementator with regard to a structure and operation of the "mechanism" of multidimensional quantity generation. Thereby, the development of such a model is a result of synthesis of knowledge, which is far from conventional metrology.

In accordance with the T. Kuhn's theory [3], the development of science is of a discrete nature, the basis for the next "scientific revolution" (i.e., for forming a new step in the development of researches in some area) being a scientific paradigm shift. The scientific paradigm shift causes the change of accents in a corresponding field of science. According to this theory, changes in the set of ideas by which the scientific community (for example, the metrology community) is guided, inevitably result in resistance by a part of that community. This resistance is caused by a fear of losing employment or status, or by a necessity to develop new skills or higher levels of skill, etc. In order to soften the negative consequences of a "scientific revolution", it is necessary to explain its inevitability and to determine the new tasks which have to be solved. For metrology experts, the accent on measurement quantities that characterize living creatures including humans, unquestioningly, determines the "scientific" revolution.

The most important reaction to a sensory information perception is the initiation of emotions. Emotion is the

\footnotetext{
a Corresponding author: k.v.s@ vniim.ru
} 
feeling that arises in an individual in the course of cognizing the reality-life. Information received produces some emotion, and the latter transmits emotion-driven information. According to $[4,5]$, the emotions motivate, organize and guide the perception, thought, and actions. Emotions are perceived and communicated mainly with the help of tactile, acoustic, visual and other impacts. For example, the taste is defined on the basis of integrating the signals received by taste gustatory receptors (buds) of various types.

To make a property measurable, it should be expressed quantitatively, i.e., in numbers with a reference. Such a reference can be a "measurement unit, measurement procedure, reference material, or combination of such" [6].

Measurement of emotions is one of the key problems of metrology in the field of studying humans. There is no generally accepted "scale of emotions". The "mechanisms" of emotion initiation have been poorly studied.

In section 2 of the paper a measurement model developed by the authors is presented. It enables signalsstimuli generating emotions in sound combinations and their series to be selected and quantitatively estimated. The measurement model is based on the analysis of the evolution of biolinguistic signals and their emotional perception. Possibilities to decode the emotional content of biolinguistic signals of animals in various situations are considered in Section 3. It is demonstrated that the model agrees with the latter investigation results concerning emotional perception of music fragments. In section 4 the results are summarized and future tasks of studies are indicated.

\section{Measurement model}

Below the acoustic method for transmitting and receiving information concerning an emotional state is considered since it is the simplest one.

According to the authors' hypothesis [7-9], any acoustical impact, carrying emotional information, contains one or a number of signals-stimuli, the perception and recognition of which in a corresponding sequence cause generation of emotions. In its turn, each of these stimuli can be characterized by one or a number of parameters. They can be measured and related to the known measurement units.

A set of these parameters characterizes peculiarities of a simple emotion, i.e., its nature, similarity to the other one, intensity, and so on. A combination of various emotions determines the variety of emotional images, while their variation with time represents an emotional content. Accordingly, the measurement of emotions stimulated by acoustical impacts can be brought to revealing experimentally the above signals-stimuli, quantitative evaluation of the parameters characterizing them and then recognition of the emotions and emotional images on the basis of the results obtained.

The model connecting the emotions and acoustic signals stimulating them is considered below. This model developing the ideas [7-9] is shown in Fig.1. The measurement model includes:

- $\quad$ adjusted time-delay circuit;

- two-channel non-linear converter;

- $\quad$ selector of sound signals determining the frequency domain;

- $\quad$ selector of signals-stimuli;

- $\quad$ analyzer, providing the identification of emotional images;

- $\quad$ associative memory of emotional images;

- comparator of emotional images and their groups.

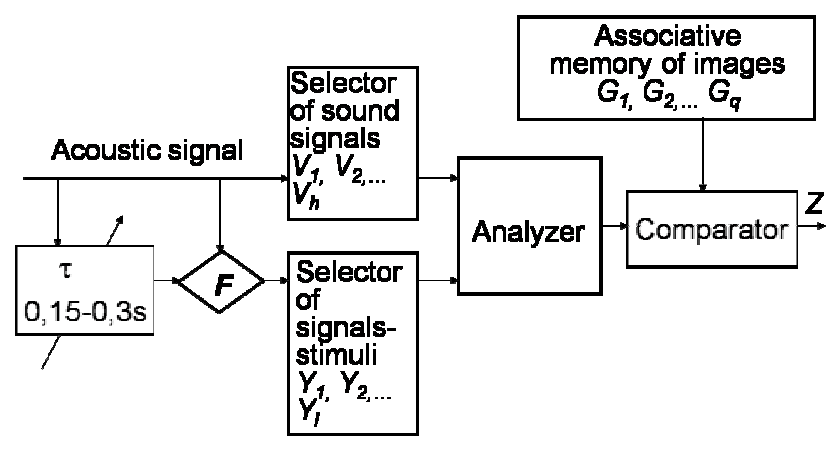

Figure 1. The model connecting the emotions and acoustic signals stimulating them

The model can be described in the following way.

$Z=Z\left[Y_{l}\left(x_{1}, \ldots x_{i} \ldots x_{r}\right), Y_{2}\left(x_{l}, x_{i} \ldots x_{r}\right), \ldots, Y_{l}\left(x_{l}, \ldots x_{i}\right.\right.$ $\left.\left.\ldots x_{r}\right), \tau, V_{l}, V_{2, \ldots} V_{h}, G_{l}, G_{2}, \ldots G_{q}\right](1)$

where

- $Y_{l}, Y_{2, \ldots} Y_{l}$ are signals-stimuli;

- $l$ is the number of various signals-stimuli;

- $x_{i}$ is a parameter measured;

- $r$ is the number of parameters;

- $\tau$ is a delay time;

- $V_{l}, V_{2, \ldots} V_{h}$ are frequency zones of sound signals;

- $h$ is the number of various frequency zones;

- $F$ is a conversion function of the non-linear converter, which is characterized by a polynomial (its degree is $\leq 11$ ) [7];

- $G_{l}, G_{2, \ldots} G_{q}$ are emotional images contained in the associative memory;

- $q$ is the number of emotional images contained in the associative memory;

- $Z$ is a complicated emotion.

To justify the measurement model (Fig.1), that reflects the "mechanism" of emotion initiation as a response to acoustic signals, sensor system evolution is briefly considered below.

Sensor systems were acquired by living creatures in ocean for stimulating the activities providing species with the ability to survive under short-term changes of the environment. The earliest sensor system provided the sense of touch, i.e., the perception of water pressure changes. The audition is its latest "modification", adapted to the perception of oscillations of air (the medium with density which is lower than that of water).

The natural selection had to lead to generating unconditioned reflexes (prototypes of the emotions) of living creatures. These reflexes relate to the responses on 
some types of perceptible changes of the environmental pressure, which cause signals-stimuli. Certainly, the frequency of perceptible changes cannot exceed a cutoff $(500 \mathrm{~Hz})$ of signal transmission over a nerve fiber. In fact, the frequency range of perceptible pressure changes is significantly lower. It can be conditionally limited by approximately $30 \mathrm{~Hz}$. This frequency range covers an infrasound and low part of the sound range. Further on, this range will be referred as IR.

In the proposed measurement model, for IR signalsstimuli their frequency and level have been accepted as the main parameters $x_{i}$ to be measured. Later, taking into account the nonstationarity of perceived acoustic signals, the authors intend to consider the expediency of other parameters characterizing the intensity and changeability of signals-stimuli.

It is well-known that animals and men perceive the IR waves as those which carry emotions [10 - 13]. The creatures living in air perceive informative IR waves both by

- body (directly) using tactile sensation of the mechanical IR waves and

- "ear-brain" system (indirectly) perceiving the air waves of the sound frequency range and then recognizing the corresponding IR waves through a special "mechanism".

The earliest emotions, e. g., fear, were negative. They were caused by a danger threatening to an individual organism. The genesis of a community gave rise to a need for transmitting information that was important for survival of the species to other members of the community. This information could be connected with either negative emotions or positive ones (e. g., availability of food).

This assumption agrees with the investigations performed by Jurgens, Blumstein and others, mentioned in the review [14], where it is proved that the majority of mammalian sounds are caused by emotions, the greater part of them being connected with a negative emotional state. In those cases when an animal experiences positive emotions, it vocalizes more rarely.

Development of the human society is connected with a greater differentiation of situations and more developed palette of emotional reactions.

The number of IR wave parameters being extremely restricted, the enrichment of emotional information was achieved by forming the emotional images on the basis of small ensembles of signals-stimuli and then taking combinations of such images in some sequence.

Such a structure resembles a speech structure with a limited number of sounds, wide set of words and unlimited possibility to transfer substantial information.

The authors explain the "mechanism" of selection of the IR oscillations from the acoustical ones, by the following way. In the "ear - brain" system, a non-linear conversion of input sound signals is performed, which results in forming the IR intermodulation products with frequency $f_{k}$ and order $p_{k}$. The level of an intermodulation products rapidly, but irregularly decreases with the growth of $p_{k}$. since it depends on the $p_{k}$ value as well as on the fact whether $p_{k}$. is even or odd. Under the conversion of the input sound signals they can be added to the same, but delayed signals.

$$
\begin{gathered}
f_{k}=\left|\sum_{i=1}^{m} n_{i} f_{i}\right| \\
p_{k}=\sum_{i=1}^{m}\left|n_{i}\right|
\end{gathered}
$$

where

- $n_{i}$ is any integer (positive or negative one, or $n_{i}=0$ ),

- $\quad m$ is the number of interacting signals with various frequencies.

After selection of the signals-stimuli, identification of their ensembles and recognition of the emotional images are realized, the simplest emotional image being formed usually on the basis of three IR signals-stimuli.

It should be noted that the non-linearity of the acoustic wave conversion in the "ear-brain" system is noticed by many authors [15-17], but it has not been studied well. In particular, the non-linearity of wave conversion for man is confirmed by:

- formation of both the microphone potential, repeating the sound wave form, and the so-called summation potential, tracing the form of sound wave envelope, in the ear cochlea;

- residual effect providing the possibility to select a pitch of waves, including IR ones, at the perception of a number of their harmonics by ear, as well as to "hear" the sound corresponding to the difference of wave frequencies with the maximum level at the impact of a number of waves with aliquant frequencies;

- dependence of the amplitude-frequency characteristic of the "ear-brain" system on the amplitude of an acoustic impact;

- $\quad$ self-adjustment of the nearest critical band (one of 24 bands which provide the sound distinguishing by frequency) to the frequency of an audible acoustic sound signal;

- finally, the fact that the "ear - brain" system is the dynamic system with variable parameters, its adjustment being realized directly in the process of audition.

Availability of adjusted delay (short-time memory) in the "ear - brain" system and joint conversion of directly effecting audible sounds and retained sounds enable a man to select corresponding signals-stimuli and perceive an emotional colour of a melody. As it follows from a further analysis of biolinguistic signals, a value of this delay corresponds to the duration of generating the simple emotions (for a man it is about 0.15-0.3 s [18]). To identify the emotions of a later origination in biolinguistic signals of mammals, including human beings, the selector of sound frequencies was included in the measurement model (Fig.1).

Each of the frequency zones has some advantages for transmitting the signals with a special emotional colour. For example, an increase in biolinguistic signal frequency increases the radius of its propagation [9]. Accordingly, the transmission of an alarm signal at a high frequency contributes to the survival of a species. The threat signal, 
as a rule, has a close addressee, the transmission of the latter being more effective at a low frequency.

Emotional colour of signals-stimuli, that living creatures acquire during the evolution process, can be identified on the basis of the relation between emotional states and activated brain biorhythms of a man. The most representative data are given in Table 1.

Table 1. State of a wakeful man and activated brain biorhythms (a cutdown version)

\begin{tabular}{|c|c|l|}
\hline Bio-rhythm & $\begin{array}{c}\text { Frequency } \\
\text { range, } \mathrm{Hz}\end{array}$ & State of a wakeful man \\
\hline$\delta$-rhythm & $0.5-4.0$ & Some types of a stress \\
\hline$\theta$-rhythm & $4.0-8.0$ & Generation of bright images \\
\hline$\alpha$-rhythm & $8.0-12.0$ & $\begin{array}{l}\text { Increse of sensibility and } \\
\text { muscular activity }\end{array}$ \\
\hline$\beta$ - rhythm & $12.0-29.5$ & Emotional excitement \\
\hline
\end{tabular}

\section{Analysis of biolinguistic signals}

The analysis was carried out on the basis of descriptive information and records of biolinguistic signals [19-23].

One of the oldest species of invertebrate living creatures is crustacea. They inhabit ocean in the form of big groups. To provide the survival of the species, they require communicating. At any excitation they emit sounds of the same type, which have different intensity depending on a situation. For shrimps (Alpheidae), these sounds are separate snaps of duration less than $0.1 \mathrm{~s}$, produced by claws. They are packs of damping waves with the frequency no less than $10 \mathrm{kHz}$. For spiny lobsters (Palinuridae), these packs are formed by sharp "flashes" of the chirring with the frequency up to some $\mathrm{kHz}$, which are evoked by rubbing feelers (antennas) [20, 22]. The intervals between these packs vary from a few milliseconds to some tens of seconds.

On the basis of the data given it is possible to come to a conclusion that the signals with a "proto-emotion" have not been differentiated with regard to amplitude and frequency for the invertebrates considered. This is the excitation which corresponds to threat, fear, or appeal of a female. In essence, such signals are signals of stress. They are packs of waves with carrier frequency sufficient for communication of information to a rather long distance, i.e. to a rather great number of crustacea. Thereby such signals contribute to the survival of species. However, tactile perception of the pulses of such a carrier frequency is impossible. It follows from this statement that the reception of such signals requires the non-linear conversion, i.e., the detection of them with selecting the energy in the lower part of the IR, which can be conventionally related to the field of the $\delta$ - rhythm.

The analysis of the biolinguistic signals provides the possibility to single out from the crustacea those who have made the next step upwards in the evolution of the nervous system.

One of the crustaceous species, crab Uca annulipes, emits sound series (packs) by knocking at the frequency up to $10 \mathrm{~Hz}$ with a claw (the duration of the pack is $1-3$ $\mathrm{s})$. If the crab is lighted up at night, it emits a knock with the frequency of $5-7 \mathrm{~Hz}$, but the duration of this knock is of several minutes [20]. Thus, the "proto-emotions" of the Uca annulipes are differentiated: in various situations the crab generates signals with the frequency of the $\delta$ and $\alpha$ - or $\theta$-rhythm, qualitatively distinguishing by emotional colour.

Fishes, the vertebrates, who inhabit the ocean too, use, at least, signals of two types: separate packs containing waves with different frequencies for various types of fish (similar to a creak, cronk, or drum roll, etc.) and long-term series of such packs. It is possible to meet with mention of signals of the third type, i.e., the whistle. Each of these signal types corresponds to a definite situation, for example, the series are typical for spawning, while irregular packs (signals with the frequencies of the $\delta$-rhythm) are characteristic of the danger sprung up.

A variety of the signal types and, accordingly, a set of corresponding main emotions grow with the nervous system evolution and achieve the maximum for the mammals.

The number of the signals associated with stress, including danger or threat, should be complemented with irregular hisses emitted by reptiles, birds, e. g., geese, swans, owls, as well as a number of mammals such as beavers, rats and leopards. The predators can emit other threat signals, e. g., growling and howling, which are typical for leopards and wolves, at the same time the leopards use the hisses too. All these sound versions can also be represented as packs of waves forming the various signals-stimuli, including signals of the $\delta$-rhythm, after the non-linear conversion in the "ear - brain" system.

If single or rare sounds generate the signals-stimuli in the lower part of the IR, then the biolinguistic signals connected with the positive emotions, should have energy splashes in the upper frequency part.

For an experimental analysis of biolinguistic signals, the authors applied software "Music 1.0" developed at the D.I. Mendeleyev Institute for Metrology. This software provides the possibility to analyze the spectrum of the IR waves formed after the non-linear conversion of the acoustic fragment or its sum with the same but delayed fragment. When the authors examined musical fragments [8, 9], they mentioned the usefulness of converting just the sum of such a type in measuring the emotions. But in analyzing the biolinguistic signals the efficiency of the model with the delay appeared to be particularly noticeable. When the delay is close to a latent period of emotion formation (about $0.2 \mathrm{~s}$ ), on the spectrograms of IR waves, obtained after non-linear conversion, the zones of energy splashes are more distinct. Just this version of the model has been used for further experiments.

Examples of results obtained are given in Fig 2 and 3. The $x$-axis scale for spectrograms is formed by the software automatically. To some extent, the $x$-axis scales are different for various spectrograms, since signal fragments that were analyzed had various duration.

It should be emphasized that due to the imperfection of the "Music 1.0" software the spectrograms of IR waves indicate a decrease from the maximum (1.0) in $\delta$ rhythm zone (as a rule, $0.5-1.0 \mathrm{~Hz}$ with the measurement time used for the analysis). Extension of the zone of the 
decrease or existence of a supplement picks in it, points to the presence of a signal-stimulus corresponding to a threat or fear.

A spectrum analysis of a howl, growling and hiss of a cheetah (acinonyx jubatus) after the square-conversion of input and delayed signal sum (Fig. 2 a-c) was carried out. For all the signals it has demonstrated an increased level of spectrum level in the bottom zone of the $\delta$-rhythm as well as in the $\theta$ - and $\alpha$-rhythms.

However, for the howl (Fig. 2 a) the energy of a signal-stimulus of the $\delta$-rhythm is concentrated near the frequency of $1.5 \mathrm{~Hz}$. At the same time; the levels of the signals-stimuli are close to each other in the zones of the $\theta$ - and $\alpha$-rhythms. The energy in the $\delta$ - и $\theta$ - rhythm zones are comparatively small (it is concentrated in a relatively narrow area). The increase in the $\beta$-rhythm zone is insignificant. The maximum energy is in the $\alpha$-rhythm zone.

If one accepts that the relations between the psychic state of a wakeful man and activated brain biorhythms (Table 1) can be extended to animals, cheetah's emotions can be decoded in their following way. The level of threat is comparatively small, but a muscular activity, to some extent, is increased. The howl is functionally connected with an addressless "claim" of the cheetah to be the master of a territory [24]. However, from the character of the biolinguistic signal it is possible to conclude that this "right" is declared only to other predators that are not large in size.

At a growl (Fig. 2 b) the picture is different. The energy in the zones of the $\delta$-, $\theta$ - and $\alpha$-rhythms increases noticeably. This means that the threat is not a "declaration". It is directed to a certain address, muscles are toughened, but the emotional excitement is not distinctive: a potential conflict can be solved without force application.

At a hiss (Fig. 2 c), which follows the growl, the energy in the $\beta$-rhythm zone grows up additionally, the emotional excitement becomes stronger, a lunge on an enemy is probable.

It is typical that in [24] both last vocalizations are referred to the aggressive ones. At the same time, the fact is emphasized that both growling and hissing are in many cases accompanied by threatening poses.

A howl of a wild dog (dhole, Cuan alpinus) presented in Fig. $2 \mathrm{~d}$ reminds the howl of cheetah (Fig. $2 \mathrm{a}$ ). However, the energy of signals-stimuli in the zones of the $\delta$ - and $\theta$-rhythms is higher. Therefore, "positioning" as a "master" is expressed clearer.

In the converted spectrum of a scream of a yellow ground squirrel (Spermophilus fulvus), taken out of a hole (Fig. 2 e), the stress of the latter can be clearly seen. Almost the whole energy splash covers the $\delta$-rhythm field and lower part of the $\theta$-rhythm field.

The converted spectrum of cheetah female purring in a comfort state (Fig. 3 a) is of another quality. The main energy is concentrated in the zones of the $\theta-, \alpha$ - and $\beta$ rhythms. The energy peak is close to $25 \mathrm{~Hz}$ (it is not shown in Fig. 3 a). The decoding can be as follows: the animal is excited, bright images present in its "imagination"; it enjoys her feelings and "listens" to them.
The converted spectrum given in Fig. $3 \mathrm{~b}$ corresponds to the peaceful interaction of wild dogs in a group. The maximum part of energy is concentrated in the field of the $\theta$-rhythms and the noticeable upraises are in the zones of the $\alpha$ - and $\beta$-rhythms (in the zones of $12-16 \mathrm{~Hz}$ and $25 \mathrm{~Hz}$ ). Possible decoding: vivid images, muscle activity and increased emotional excitation peculiar to playing.

The carrier frequency of mammal sounds demonstrates whether a stress is caused by fear or due to an aggressive threat addressed to another animal. Therefore, in the above measurement model the selector of sound signals was included, which enables the emotion identification to be detailed. The fear signal is emitted at a high frequency that is maximal for an animal in order to transmit it to a greater number of animals of the same species. The signal of threat addressed to the animal that is nearby, is transmitted at a low frequency of sounds, because it is not necessary to pass this signal to other animals. An addressless threat signal, howl, is transmitted at the frequency of the medium sound range.

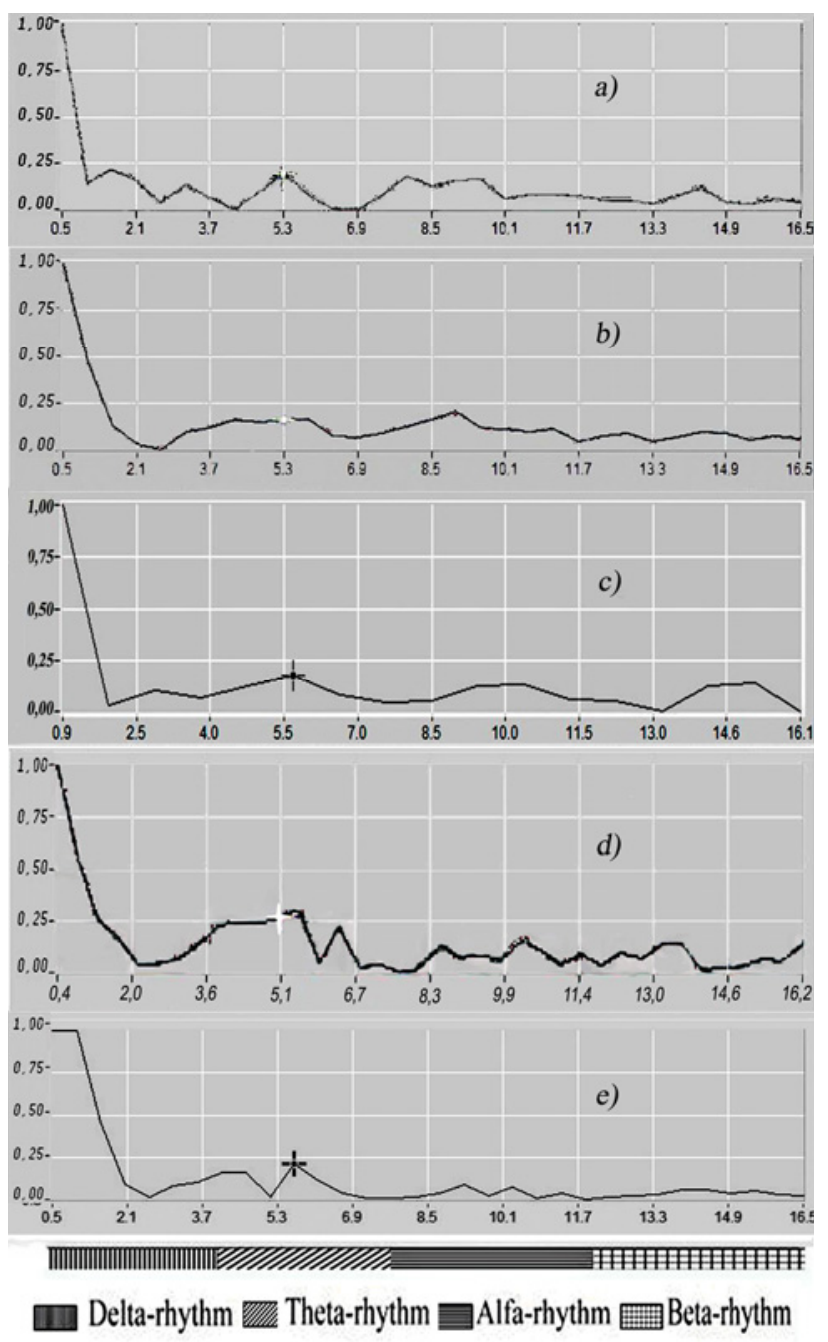

Figure 2. Spectra of the IR intermodulation components after non-linear conversion of the input data ( $x$-axis is the frequency, $\mathrm{Hz} ; y$-axis is the level of spectrum components determined in conventional units)

a) cheetah howl; b) cheetah growl; c) cheetah hiss; d) wild dog howl; e) scream of a yellow ground squirrel, taken out of a hole 
It should be noted that the model used also allows the emotional colour of the biolinguistic sound signals of living creatures to be measured independently of their mass and dimensions, range of the frequency of the sounds they emit and even of the level of living creature development or complexity of its apparatus emitting sounds.

The analysis presented enables some remarks to be made relating to the concepts accepted in biology which explain the peculiarities of biolinguistic sound signals. In particular, in [25] it is stated that the resemblance of hissing and growling as well as of other signals emitted by different animals, can be explained by imitation of sounds (onomatopoeia). In $[14,26]$ an idea is put forward that the estimates of the emotion excitation obtained on the basis of energy parameters and shift of the acoustic energy to the high frequency field of the spectrum are the most reliable ones and, consequently, just these parameters of biolinguistic signals contribute to mutual understanding of men and animals.

In the opinion of the authors of the present paper, both the similarity of sound signals and ability of different species to communicate with each other are determined by the same unified mechanism of perception of the biolinguistic signal emotional colour, which was originated in the process of evolution. The decoding of emotional colour depends on the parameter of signals in the sound range and in the IR, the information value of the IR being higher.

Information given above is in accordance with [7-9] where it is shown that the emotional colour of music is determined by the IR oscillations. In particular, the difference in emotional perception of the major and minor tonalities corresponds to the difference in the spectrum of the intermodulation products. The simplest emotional images are formed by sequences of the attendant tonality chords and so on.

The analysis of the ethnic (African) music with a known emotional mood has shown that it is possible to decode it by identification of the simplest ensembles of signals-stimuli, taking into account information of Table 1on the relation of the state of a wakeful man and activated brain biorhythms connected them with the $\delta$-, $\theta-, \alpha-$ and $\beta$ - rhythms of a man.

These results enable one to state that the mechanism of emotion generation has improved in the process of development of human civilization, but it has not cardinally changed.

Thus, the proposed measurement model reflects consistent patterns of biological evolution of emotional perception related to acoustic impacts. It provides a possibility to decode emotions generated when listening to music fragments or sounds emitted by living creatures.

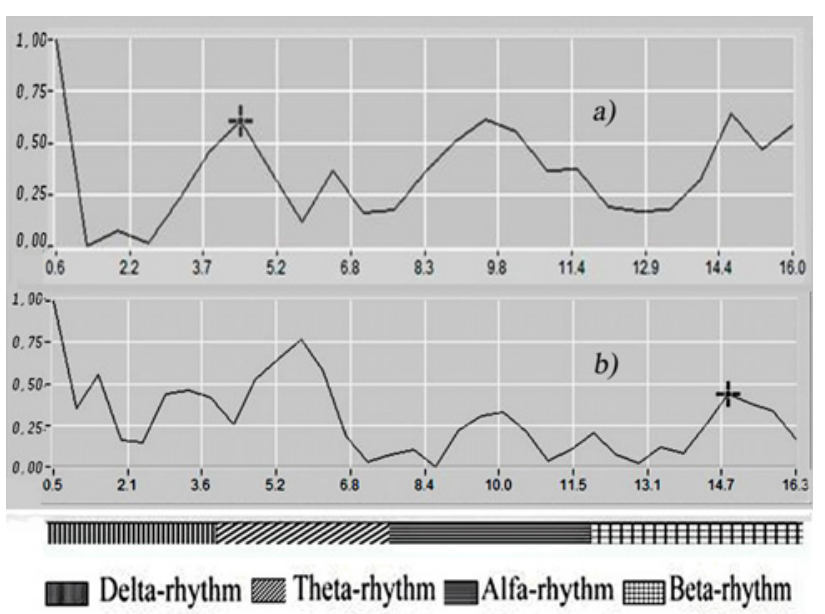

Figure 3. Spectra of the IR intermodulation components after non-linear conversion of the input data ( $x$-axis is the frequency, $\mathrm{Hz} ; \mathrm{y}$-axis is the level of spectrum components determined in conventional units)

a) cheetah female purring; b) peaceful interaction of wild dogs

\section{Conclusions}

The measurement model considered above, which is intended for measuring the emotions initiated as a result of listening to acoustic impact, reflects the regularities common for living creatures. The correspondence of the model to the "mechanism" of emotional reaction generation has been grounded by

- analysis of development of this "mechanism" in the evolution process from invertebrates to humans;

- results of application the measurement model for investigation of the emotional colour of biolinguistic signals;

- concordance of the latter investigation with the results of the studies of emotional perception of music fragments carried out earlier.

The model proposed opens new lines for investigations in biology, ethology, psychology, musicology, and in a number of technical sciences.

The list of main problems connected with the uncertainty of results obtained in measuring the emotions should include the insufficient knowledge of:

- relationship of the neuro-process frequencies, particularly, the brain bio-rhythms frequencies, and mood;

- scale of brain biorhythms taking into account biorhythm subzones;

- $\quad$ boundaries between different emotions;

- influence of genetic predisposition, psycho-type, upbringing and cultural traditions on generation of the emotional response to acoustical signals.

The nearest perspectives of applying the model, to the authors' opinion, are mostly noticeable in the fields connected with

- development of methods and means of diagnostics of human psyche development, including dynamics of infant's psyche development;

- $\quad$ purposeful influence on the emotional sphere of an animal or man; 
- $\quad$ theory of music and musical instruments;

- automatic interpretation of emotionally coloured speech.

It should be also said that the approach to development of the measurement model, the authors have created, can be extended to the sphere of receiving transmitting emotionally coloured information of a visual nature.

\section{References}

1. K. Sapozhnikova, A. Chunovkina, R. Taymanov, Measurement, 50, pp. 390-396 (2014), DOI: 10.1016/j.measurement.2013.06.026.

2. A. Chunovkina, K. Sapozhnikova, and R. Taymanov, Proceedings of they XX IMEKO World Congress, September 9-14 2012, (Busan, Republic of Korea, 2012) $6 \mathrm{p}$.

3. T.S. Kuhn, The Structure of Scientific Revolutions (University of Chicago Press, Chicago, 1962).

4. C.E. Izard, The Psychology of Emotions (Springer US, 1991).

5. G.A. Golitsyn, Information and Creativity (Russkiy Mir, Moscow, 1997).

6. International Vocabulary of Metrology - Basic and General Concepts and Associated Terms, 3rd edition, 2008 version with minor corrections, (BIPM, JCGM 200, 2012).

7. K. Sapozhnikova, R. Taymanov, Proceedings of the XVII IMEKO World Congress, 22-27 June 2003 (Dubrovnik, Croatia, Croatian Metrological Society) pp.2049-2053, http://www.imeko.org/

8. K. Sapozhnikova, R. Taymanov, Proceedings of the ISMTII'2009, 29 June - 2 July 2009, (St. Petersburg, Russia, 2009) pp. 4-239- 4-243.
9. R. Taymanov, K. Sapozhnikova, Meas Sci Rev, 10, 3, pp. 78-88 (2010).

10. W. Seabrook, Doctor Wood. Modern Wizard of the Laboratory (Harcourt: Brace and company, New York, 1941).

11. Experiment: Conversations in Art and Science, eds. B. Arends, D. Thackara (The Welcome Trust, 2004).

12. A. Kaczmarska, L. Uczak, Archives of Acoustics, 33, 3, pp. 331-340 (2008).

13. H. Yuan, H. Long, X. Mou, Chinese Journal of Rehabilitation Medicine, 23, 5, pp. 385-387 (2008).

14. I.A. Volodin, E.V. Volodina, S.S. Gogoleva, L.O. Doronina, Journal of General Biology, 70, 3, pp.210-224 (2009).

15. V.K. Labutin, A.P. Molchanov, Mechanism Audition Models (Energia, Moscow, 1973).

16. I. Aldoshina, R. Pritts, Musical Acoustics (Kompozitor, St. Petersburg, 2006).

17. C.U.M. Smith, Biology of Sensory Systems (Chichester, John Wiley \& Sons, 2000).

18. O. Varyagina, A. Khromatidi, E. Bratico, N. Novitsky, M. Tervaniemi, Sensornye Sistemy, 20 3, pp.180-186 (2006).

19. http://www.bioacoustica.org/gallery/gallery_rus.html 20. N.I. Tarasov, Alive sounds of sea (AN SSSR, Moscow, 1960).

21. Infrasound http://andreyrazdrogin.narod.ru/ infzvuk.html

22. http://www.zooeco.com/0-dom/0-dom-r2-18-10.html

23. http://dic.academic.ru/dic.nsf/enc_biology/166/

24. E.V. Volodina, Zoological Journal, 79 7, pp. 833-844 (2000).

25. O.L. Silaeva, Onomatopoeia: Science and Practice (Moscow, 2008).

26. P. Pongracz, C. Molnar, A. Miklosi, Appl. Anim. Behav. Science, 100 3-4, pp. 228-240 (2006). 